\title{
Site-centered impurities in quantum spin chains
}

LPENS-Th-07/2001

\author{
P. Pujol and J. Rech \\ Laboratoire de Physique, Groupe de Physique Théorique \\ ENS Lyon, 46 Allée d'Italie, 69364 Lyon Cédex 07, France.
}

\begin{abstract}
The magnetic behavior of antiferromagnetic spin $1 / 2$ chains with site-centered impurities in a magnetic field is investigated. The effect of impurities is implemented by considering different situations of both diagonal and off-diagonal disorder. The resulting magnetization curves present a wide variety of plateaux, whose position strongly depends on the kind of disorder considered. The relevance of these results to experimental situations is also discussed.

PACS numbers: 75.10.Jm, 75.10.Nr, 75.60.Ej.
\end{abstract}

\section{INTRODUCTION}

The study of the magnetic properties of low dimensional antiferromagnets has received much renewed attention during the last years. One particular issue that captured both experimental and theoretical efforts is the appearance of magnetization plateaux in spin chains and ladders systems. In general, those plateaux appear for pure spin systems at rational magnetization values 11 . Some experiments have indeed confirmed these theoretical predictions in a few particular cases [5].

More recently, the effect of the presence of impurities on such magnetic behavior was also investigated. From the theoretical point of view, the properties at zero magnetic field have largely been elucidated [6,7], and recent analysis explored also the robustness of the rational plateaux for small amplitude of the disorder [8]. A very interesting phenomenon recently discovered was also that, for bond-disorder with discrete probability distributions, plateaux at non-rational values of the magnetization are present [9]. Moreover, the position of such plateaux in the curve is related in a simple way to the concentration of impure bonds in the system. Since this kind of disorder is a good candidate for modelling concrete experimental situations, this result opens new perspectives in the interpretation of experimental curves. In order to make closer contact with experiments, one has to take into account all the effects that can be produced by non-magnetic impurities, and provide a wide range of possibilities for implementing the presence of such impurities in realistic models.

In this paper we analyze a simple model where different kinds of diagonal and off-diagonal disorder are present. The techniques used are based on a decimation procedure [10], as well as standard quantum mechanics tools and numerical calculations for $X X$ chains of sizes ranging from 50000 to 100000 sites. Despite the simplicity of our model, we find that a huge number of families of cases is present, each of them presenting its own hierarchy of magnetization plateaux. Moreover, we show how a simple tuning of the microscopic parameters can drive our system from one family to another. We also argue that this simple example serves as a good laboratory for understanding and classifying the wide variety of cases one can encounter in experimental curves of antiferromagnetic systems with impurities.

\section{THE MODEL}

The model we are going to study is a spin- $1 / 2$ chain whose Hamiltonian is given by:

$$
H=\sum_{i}\left[J_{i}\left(S_{i}^{x} S_{i+1}^{x}+S_{i}^{y} S_{i+1}^{y}+\Delta S_{i}^{z} S_{i+1}^{z}\right)+h_{i} S_{i}^{z}\right]
$$

The randomness is implemented by considering $J_{i}$ and $h_{i}$ as random variables. Specifically, bond randomness will be obtained by assigning to all the variables $h_{i}$ the same value fixed at $h$, and by giving a probability distribution to the set of variables $\left\{J_{i}\right\}$. The probability distribution can, of course, be chosen in such a way to give, on average, a periodicity of $q$ to the bond variables. It is important to say that realistic distributions relevant for many possible experimental situations are discrete. In this sense, a binary distribution captures the essential characteristics of the phenomena we want to describe here, the generalization to more complicated discrete distributions being straightforward. In [9], a distribution of the form:

$$
P\left(J_{i}\right)=p \delta\left(J_{i}-J^{\prime}\right)+(1-p) \delta\left(J_{i}-J-\gamma_{i} J\right),
$$

was considered, where $\gamma_{i} \equiv \gamma,(-\gamma)$ if $i=q n,(i \neq q n)$. In order to match the behavior of a disorder originating from a site impurity, a most appropriate distribution can be given by the following algorithm: distribute first regular values for the bonds $J_{i}$ with the desired periodicity $q$, and parameter $\gamma$, then, to each site of the chain assign a probability $1-p$ to be an "original" site, and $p$ to be an "impure" site. Finally, for each site $i$ which turned out to be impure, change the values of the surrounding bonds $J_{i}$ and $J_{i-1}$ to $J^{\prime}$. We are, as in [9], concerned with three 
different values for the bond strength, $J \pm \gamma J$ and $J^{\prime}$, but now with a correlated probability distribution.

For site-centered disorder, we are going to consider two different cases. The first is just obtained by writing $h_{i}$ as $h+h_{i}^{\prime}$ where $h_{i}^{\prime}$ is a random variable taking values of 0 with probability $p$ and $h^{\prime}$ with probability $1-p$. This case can be considered as academic, since it violates the symmetry $h \rightarrow-h$, but it will nevertheless provide useful insight for more realistic disorders. The second and more realistic case is the $h \rightarrow-h$ symmetry preserving case where $h_{i}=h\left(1+\alpha_{i}\right)$, with values for $\alpha_{i}$ being binary distributed among 0 and $\alpha$ with probability $p$ and $1-p$, and $\alpha \geq-1$ (the limiting case of $\alpha=-1$ corresponding to an impurity which does not couple to the magnetic field).

In the case of $\Delta=0$, the model can be mapped, via the well known Jordan-Wigner transformation, to a problem of free fermions, whose first quantized Schrödinger-like equation is:

$$
J_{i-1} c_{i-1}+J_{i} c_{i+1}=h_{i} c_{i} .
$$

The magnetization is simply related to the number of states occupied and the $z$ component of the susceptibility is just proportional to the density of states. For a given energy, the number of states can be simply obtained by the node counting method [11], related to the number of positive "self-energy" variables:

$$
\Delta_{i}=c_{i-1} J_{i-1} / c_{i}
$$

The recursive formula for these variables is:

$$
\Delta_{i+1}=J_{i}^{2} /\left(h_{i}-\Delta_{i}\right)
$$

which, for the case of bond and $h \rightarrow-h$ symmetry preserving site disorder can be written as:

$$
D_{i+1}=W_{i}^{2} /\left(h-D_{i}\right)
$$

where $D_{i}=\frac{\Delta_{i}}{1+\alpha_{i}}$ and $W_{i}^{2}=\frac{J_{i}^{2}}{\left(1+\alpha_{i}\right)\left(1+\alpha_{i+1}\right)}$. Note that $D_{i}$ has the same sign as $\Delta_{i}$, then the node counting can be done in the $D$ variables. This remark will be very useful when discussing the presence of plateaux and the behavior of the magnetization curve close to $h=0$.

\section{OFF-DIAGONAL DISORDER}

As mentioned above, this case corresponds to a generalization for bond-disorder considered in [9]. In this case each time a site is considered as an impurity, it must be surrounded by bonds with lower values. The decimation procedure used in 9] is again well adapted for identifying the location of magnetization plateaux. However, some care has to be taken in counting the number of bonds for each decimation step, particularly in the case of two impurities sitting in neighboring sites. We refer to the reference mentioned above for the details of this reasoning and present here the result for a dimerised chain which is the most relevant case for experimental situations, the generalization to generic periodicity of the lattice being straightforward. The principal plateau is located at:

$$
M=2 p-p^{2}
$$

coming from the decimation of the bonds with the highest value. The second step of the decimation gives a secondary plateau at:

$$
M=2 p-2 p^{2}+2 p^{3}-p^{4} .
$$

These plateaux are well observed in the numerical curves of figure (11), where we show the result for an $X X$ chain.

The behavior of the magnetization for small magnetic fields can also be obtained as a generalization of the normal off-diagonal disorder. For example, for the $X X$ case, a mapping to a random walk in the self-energy variables shows, as for a standard dimerised chain with random bonds [12,8], a power law behavior for the magnetic susceptibility for even periodicity of the lattice:

$$
\chi_{z} \propto H^{\lambda-1}
$$

where the exponent can be easily obtained from the mean and variance of $\ln (J)$ (see for example [9]). Using the same method, a logarithmic behavior for the susceptibility is obtained for odd periodicity of the lattice:

$$
\chi_{z} \propto \frac{1}{H\left[\ln \left(H^{2}\right)\right]^{3}} .
$$

\section{DIAGONAL DISORDER}

We first concentrate on the naive diagonal disorder one can introduce in a dimerised chain, by supposing that a supplementary magnetic field $h^{\prime}$ at each impurity is present. While for low $h^{\prime}$ no noticeable changes at the curve occur, for strong enough values of $h^{\prime}$, we see in figure (2) the appearance of new plateaux. A simple way to understand such plateaux is by considering the strong coupling case $\gamma \sim 1$. The order zero in powers of $(1-\gamma)$ is just given by a combination of dimers which can contain two, one or zero impurities with probability $(1-p)^{2} / 2$, $p(1-p)$ and $p^{2} / 2$ respectively. It is then easy to draw the magnetization curve for each case (a three step stair like curve) and then superpose the curves with the appropriate weight. The result is compared to the numerical data in figure (3). The positions of the plateaux predicted at this order are located at:

$$
M=-1+p^{2},-(1-p)^{2}, 0, p^{2}, 2 p-p^{2} .
$$

Note in passing the lack of $M \leftrightarrow-M$ symmetry in the magnetization curve due to the very nature of the disorder. Comparing with the numerical result, one sees that some plateaux are softened due to the presence of non-zero coupling which was neglected in our procedure. The next step is as usual to turn on a non-zero value for $\gamma$ and use standard quantum mechanics perturbation 
theory (see for example 13$]$ for a similar treatment). The result is a smoothing of the jumps between the plateaux and a correction to their width which can be calculated in powers of $(1-\gamma)$. Since we are concerned with more realistic kinds of disorder these computations are beyond the scope of this work. We just mention the fact that this case leaves the $M=0$ plateau present, a result which can be also obtained for the $X X$ case by the random walk argument. We refer to [11] for the details of this mapping, and just mention here that it is easy to study the cycles of +- self energy variables for low energies. One can indeed show that, in the equivalent first quantized Schrödinger picture, and for small enough but non-zero values of the energy, these cycles are infinite indicating that the number of states remains constant in a finite interval of energy, which translate in the magnetic language into a zero magnetization plateau.

The second case is obtained by assuming that each impurity couples to the magnetic field with a factor of $(1+\alpha)$. It gives a symmetric magnetization curve by the change $h \rightarrow-h$. It is sufficient to study only the case $\alpha<0$. Indeed, the case of positive $\alpha$ is obtained by a simple re-scaling of the magnetic field and the change $p \leftrightarrow 1-p$. In the case of $X X$ chains, we can make use of the change of variables defined in (6). We have then the equivalence between the model we are considering and a system with constant magnetic field, but with a wide hierarchy of values for the bonds. The decimation procedure can then be used to predict the presence of plateaux. We have however to identify different cases corresponding to each hierarchic structure of the bonds, and these are given by the values of $\alpha$ and $\gamma$ we are considering. As already stated before, the decimation procedure is implemented by eliminating the strongest bonds, and their relative weight in the system gives the position of the corresponding plateau in magnetization. We omit the details of the combinatorics and present the results for the position of the principal plateaux for each case in table (I). Figure (4) shows also the magnetization curve obtained for one of these cases, showing a fair agreement with the decimation predictions.

Let us now analyze the behavior of the system at low magnetic fields. By using again the mapping to a random walk of the generalized Riccati variables, one could naively say that equation (6) gives rise to a behavior similar to the one obtained for an off-diagonal disorder. An important difference is however that the noise in this case is cancelled step by step, giving rise to a regular and non-random walk at large times (this can be easily seen by inspecting the form of the variables $W_{i}$ ). Then, at low magnetic fields, the behavior of our systems is asymptotically equivalent to the one of a pure chain, and for an even periodicity of the bonds shares in particular the presence of a plateau at zero magnetization. Strictly speaking, these results are valid for $X X$ chains only, since we have used the change of variables (6). We conjecture however that the qualitative behavior for the curve, and in particular, the position of the magnetization plateaux remains valid for generic $X X Z$ chains.

\section{MIXED DISORDER}

This last case can be considered as the combination of both kinds of disorder studied before. It is important to notice that the equivalence between positive and negative $\alpha$ is not valid anymore due to the extra presence of bond disorder. For the $X X$ case, it is however easy to see by means of $(6)$ and the subsequent decimation procedure, that the case $\alpha>0$ has the same plateaux as for the offdiagonal disorder discussed above. This is still the case for negative but small values of $\alpha$, since the hierarchy of coupling in the effective system is unchanged. The situation is however radically changed when $\alpha$ goes below the critical value:

$$
\alpha_{c}=\frac{J^{\prime}}{J+\gamma J}-1
$$

where the hierarchy of values for the bonds is completely changed. Figure (5) shows that crossing the critical value of $\alpha$ indeed changes completely the nature of the magnetization curve and the position of the plateaux in the curve. In that case, the strongest bonds have the values $J^{\prime} /(1+\alpha)$ and can be found in arbitrary long chains of impurities. This fact invalidates the standard decimation procedure for locating the position of the plateaux. However, one can proceed by noticing that, for values of $\alpha$ close enough to -1 , the inter-impurities bonds in the effective model are much larger than the others. One can then use a kind of strong-coupling treatment in which the zeroth order system is obtained by equating the value of the remaining bonds to zero. We are then left with a collection of decoupled spin chains with arbitrary length. For an array containing $n$ spins, the eigenvalues of the Hamiltonian are simply given by the roots of second order Chebyshev polynomials

$$
E_{p}=J_{\text {eff }} \cos \left(\frac{p \pi}{n+1}\right) ; p=1 \ldots n
$$

and $J_{e f f}$ is the value of the coupling between the spins. The magnetization curve for such a finite chain can also be easily obtained. To draw the magnetization curve for the total system one just has to superpose the curves for all possible values of $n$ with the corresponding probability of appearance $(1-p)^{2} p^{n}$. The result is shown in figure (6) where we compare the numerical result with the strong coupling computation performed up to 11th order in $p$. The appearance of a hierarchy of plateaux close to saturation predicted by the theory is clearly observable in the numerical data.

The plateau at low values of the magnetization and the jump in the magnetization at zero field is an artifact of the zeroth order approximation. This can be cured now by turning on the remaining coupling to non-zero values and treating them in perturbation theory. On what concerns the jump at $M=0$, there is however a much 
simpler way of studying the shape of the curve close to $M=0$. This is achieved again by the mapping to the random walk problem. One recovers in this way the power law or logarithmic behavior as in (9)-(10) depending on the periodicity of the bonds.

\section{CONCLUSION}

To summarize, we have shown that the magnetization curve of spin chains with different kinds of impurities exhibits a rich variety of plateaux. While the phenomenon of non-rational plateaux was already observed in a disordered case [9], we have shown in this paper with simple examples that the position of such plateaux strongly depends on the particular values of the microscopic parameters. While one can speak of a sort of universality for the position of the plateaux, depending only on the concentration of impurities and periodicity of the averaged bonds, we have clearly seen that different families of disorder produce qualitatively different magnetization curves. Moreover, within the same kind of disorder (the mixed disorder case in particular) a simple tuning of the microscopic parameters can switch the system from one "universality class" to another. This scenario teaches us that the characterization of spin chains with impurities is better understood in terms of families of disorder, at least to understand the behavior of the magnetization curve. While some results in this paper have been obtained for the $X X$ case only, it is reasonable to think that such a characterization in terms of families of disordered chains remains valid for generic $X X Z$ chains. Of particular interest is the mixed case, for which we believe the case $\alpha \rightarrow-1$ should represent the magnetization curve for materials such as $\mathrm{CuGeO}_{3}$ doped with $\mathrm{Si}$ [14. We trust this work provides the necessary tools for predicting the shape of the magnetization curve in future experimental situations, where higher periodicities than 2 are also conceivable [4].

We acknowledge B. Dujardin for fruitful discussions and D. C. Cabra and A. Honecker for constructive comments and a careful reading of the manuscript.

[1] M. Oshikawa, M. Yamanaka, I. Affleck, Phys. Rev. Lett. 78, 1984 (1997).

[2] D.C. Cabra, A. Honecker, P. Pujol, Phys. Rev. Lett. 79, 5126, (1997); Phys. Rev. B 58, 6241 (1998).

[3] K. Totsuka, Phys. Lett. A 228, 103, (1997), Phys. Rev. B 57, 3454 (1998).

[4] K. Hida, J. Phys. Soc. Jpn. 63, 2359, (1994); K. Okamoto, Solid State Comm. 98, 245 (1995).

[5] Y. Narumi et al., Physica B 246-247, 509 (1998).

[6] D.S. Fisher, Phys. Rev. B 50, 3799 (1994).
[7] R.A. Hyman, K. Yang, R.N. Bhatt and S.M. Girvin, Phys. Rev. Lett. 76, 839 (1996); P. Henelius and S.M. Girvin, Phys. Rev. B 57, 11457 (1998); F. Iglói, R. Juhász, H. Rieger, Phys. Rev. B 61, 11552 (2000).

[8] K. Totsuka, Physica B284-288, 1559, (2000); K. Totsuka, Phys. Rev. B 64, 134420 (2001).

[9] D.C. Cabra, A. De Martino, M.D. Grynberg, S. Peysson, P. Pujol, Phys. Rev. Lett. 85, 4791 (2000).

[10] S.K. Ma, C. Dasgupta and C.-K. Hu, Phys. Rev. Lett. 43, 1434 (1979); C. Dasgupta and S.K. Ma, Phys. Rev. B 22, 1305 (1979).

[11] T.P. Eggarter and R. Riedinger, Phys. Rev. B 18, 569 (1978).

[12] A.A. Ovchinnikov and N.S. Érikhman, Sov. Phys. JETP 46, 340 (1977).

[13] D.C. Cabra, A. De Martino, A. Honecker, P. Pujol, P. Simon, Phys. Rev. B 63, 094406 (2001).

[14] J.-P. Renard et al., Europhys. Lett. 30, 475 (1995); L.P. Regnault et al., ibid 32, 579 (1995); M. Fabrizio, R. Mélin, J. Souletie, Eur. Phys. J. B 10, 607 (1999).

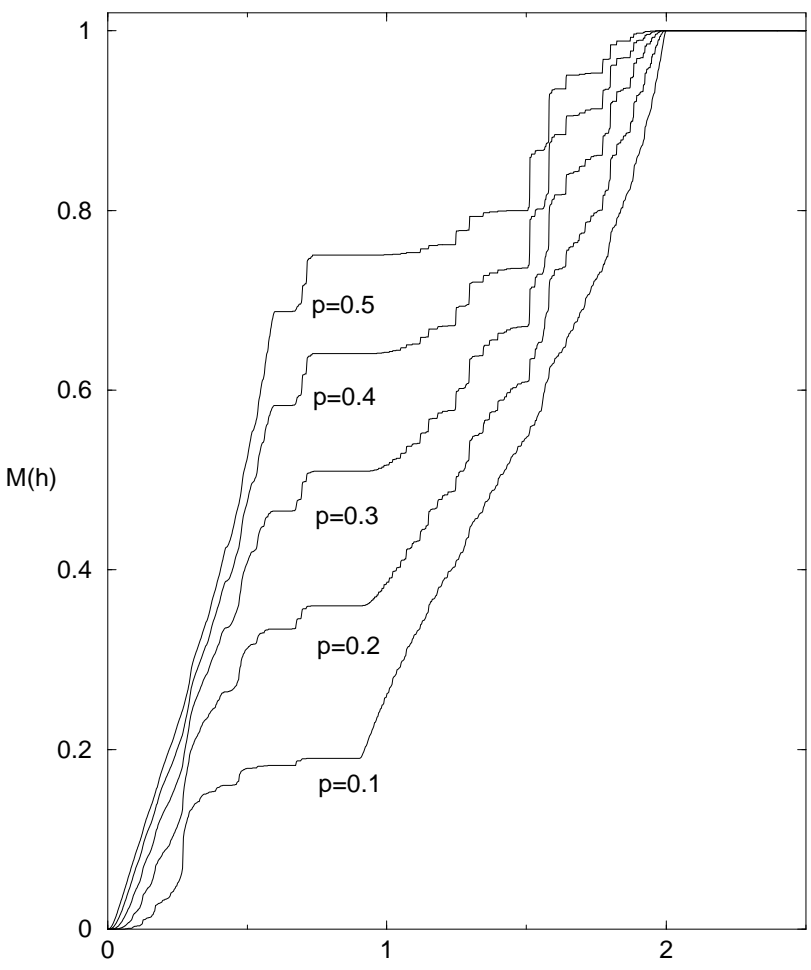

FIG. 1. Magnetization curves of $X X$ chains with off-diagonal disorder for the parameters values of $J=2$, $J^{\prime}=0.6, \gamma=0.45$. 


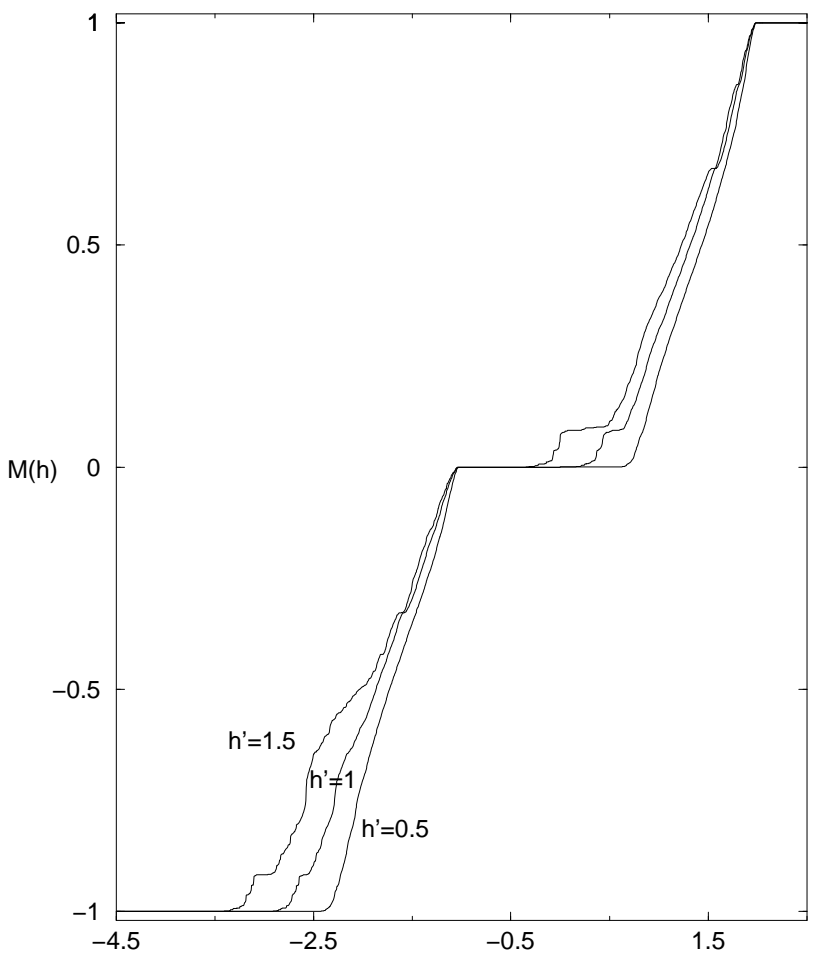

$\mathrm{h}$

FIG. 2. Magnetization curves for the first kind of diagonal disorder mentioned in the text (see eq. (11) taken with $p=0.3, \gamma=0.5, J=2$.

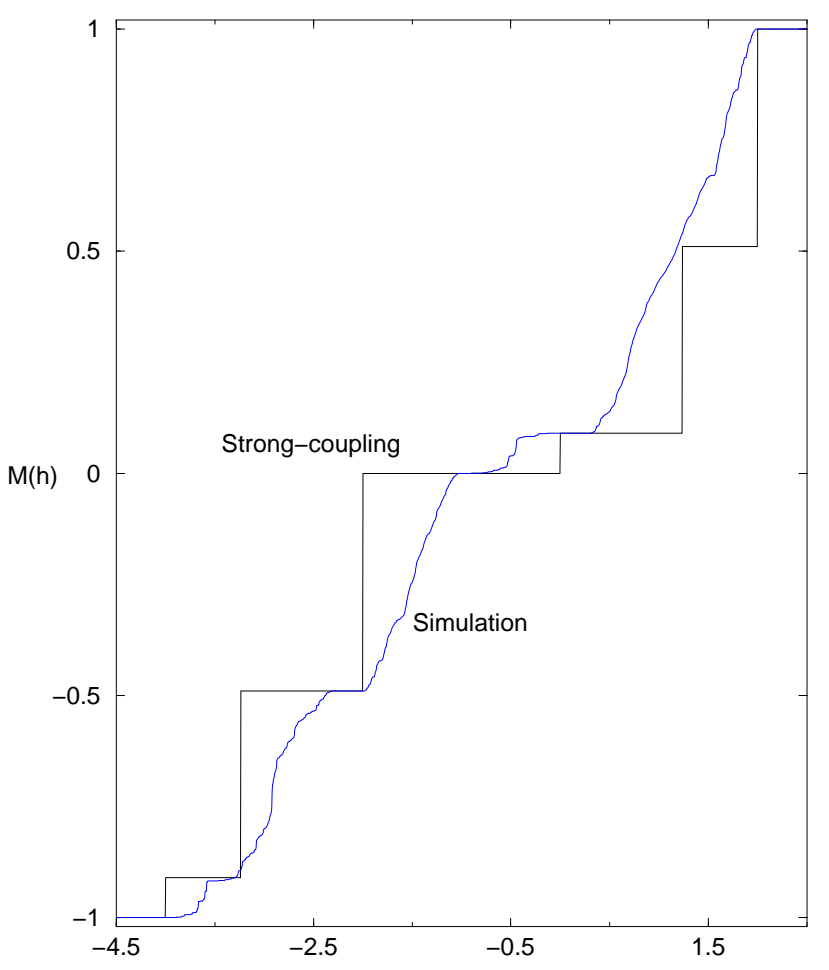

$\mathrm{h}$

FIG. 3. Same as in figure (2) with $J=2, \gamma=0.45, h^{\prime}=2$, $p=0.3$.

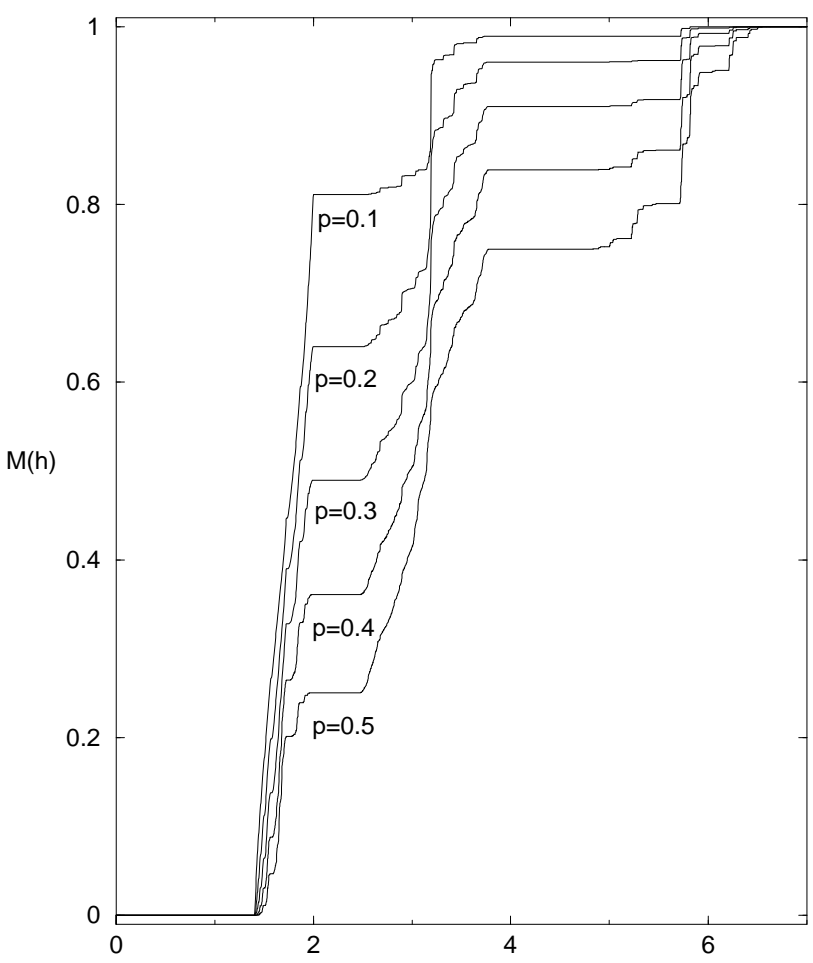

$\mathrm{h}$

FIG. 4. Magnetization curves for the $(h \leftrightarrow-h)$ symmetric disorder with $J=2, \alpha=-0.7, \gamma=0.7$.

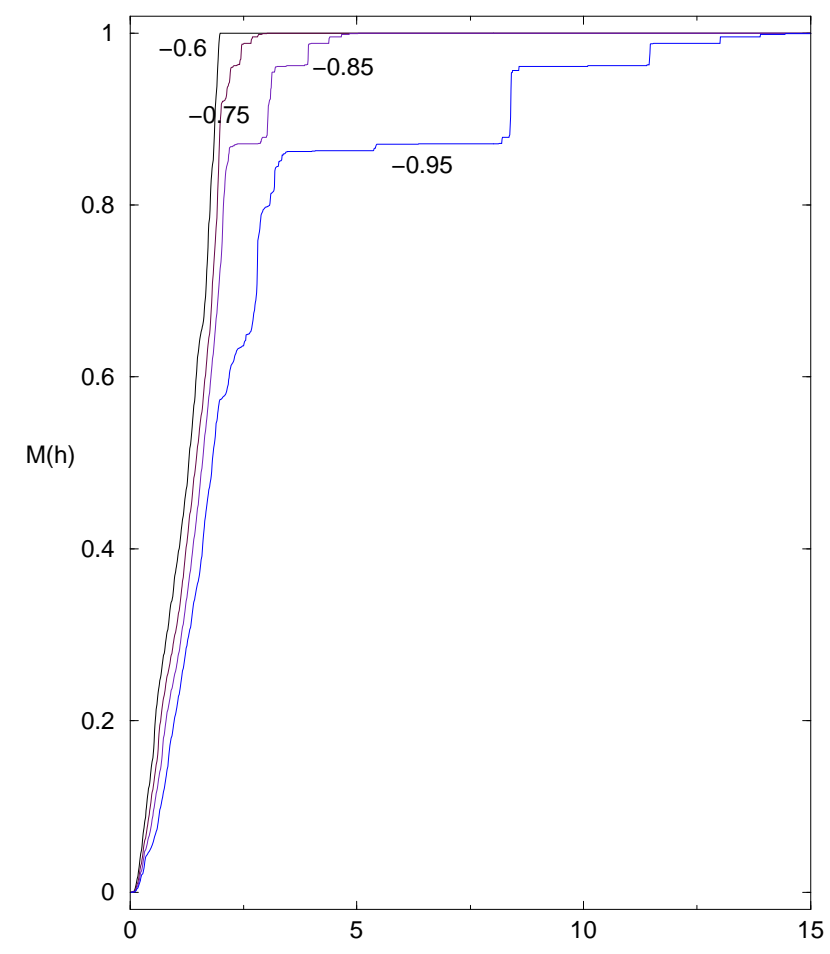

$\mathrm{h}$

FIG. 5. Magnetization curves for the case of mixed disorder with $J=2, J^{\prime}=0.8, \gamma=0.5, p=0.4$ and different values of $\alpha$. In this case $\alpha_{c}=-0.73$ (see text). 


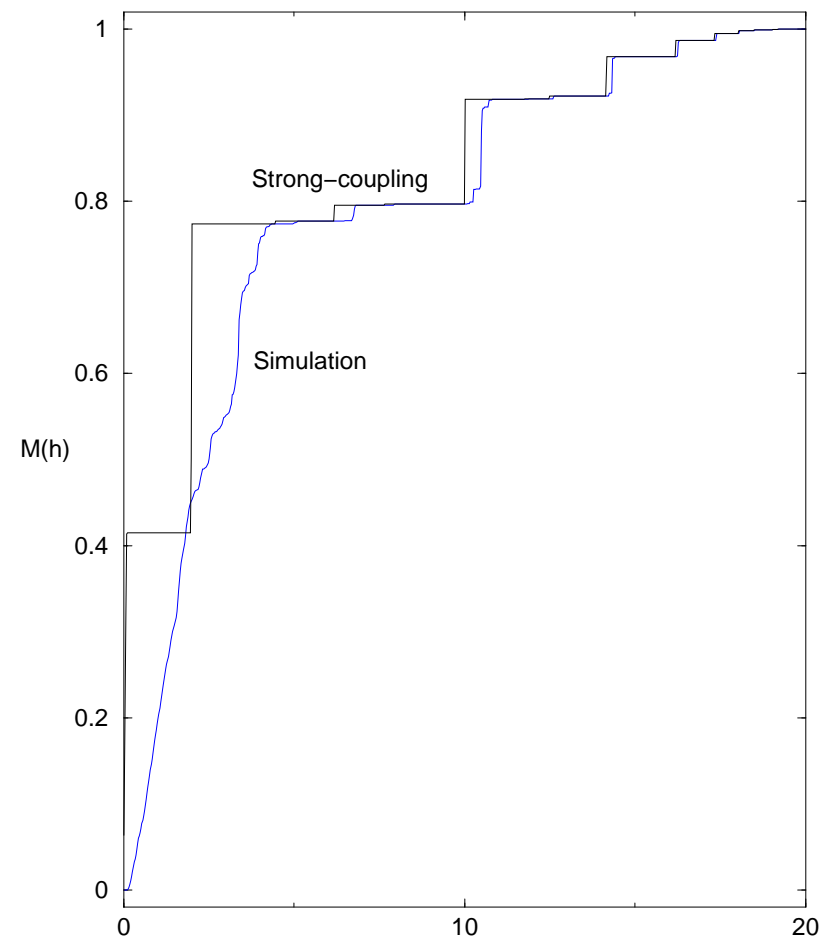

FIG. 6. Strong-coupling and numerical result for the mixed disorder case with $J=2, J^{\prime}=0.8, \alpha=-0.95, \gamma=0.5$, $p=0.3$.

\begin{tabular}{|c|c|}
\hline \hline Range of $\alpha$ parameter & Magnetization plateaux \\
\hline$\left(\frac{1-\gamma}{1+\gamma}\right)^{2}-1<\alpha<0$ & $1-p^{2}$ \\
& $(1-p)^{2}$ \\
& 0 \\
\hline$\left(\frac{1-\gamma}{1+\gamma}\right)^{2}-1<\alpha<\left(\frac{1-\gamma}{1+\gamma}\right)^{4}-1$ & $1-p^{2}$ \\
& $(1-p)^{2}\left(1+p^{2}\right)$ \\
& $(1-p)^{2}$ \\
& 0 \\
$\alpha<\left(\frac{1-\gamma}{1+\gamma}\right)^{4}-1$ & $1-p^{2}-p^{2}(1-p)^{2}$ \\
& $\left(1+p^{2}+p^{4}\right)(1-p)^{2}$ \\
& $\left(1+p^{2}\right)(1-p)^{2}$ \\
& $(1-p)^{2}$ \\
& 0 \\
\hline \hline
\end{tabular}

TABLE I. Magnetization at the principal plateaux for the $(h \leftrightarrow-h)$ symmetric diagonal disorder (see text). 